\section{Utilidad del ultrasonido endobronquial para el diagnóstico y etapificación del cáncer pulmonar}

\author{
GONZALO LABARCA ${ }^{1,2}$, IVÁN CAVIEDES ${ }^{3}$, ERIK FOLCH ${ }^{4}$, \\ ADNAN MAJID ${ }^{5}$, SEBASTIÁN FERNÁNDEZ-BUSSY ${ }^{3}$
}

\section{Endobronchial ultrasound-guided transbronchial needle aspiration}

In the last years, several techniques have been developed to obtain a prompt diagnosis or rule out lung cancer. Endobronchial ultrasound-guided transbronchial needle aspiration (EBUS-TBNA) is a minimally invasive procedure with a high diagnostic yield for mediastinal and central pulmonary lesions. This procedure is especially useful for lung cancer diagnosis and mediastinal staging. Two different types of EBUS are currently available: Radial EBUS and lineal EBUS. Each one has technical differences together with a range of clinical indications. The aim of this review is to discuss about EBUS-TBNA and its $\mathrm{cu}$ rrent clinical indications, evidence about the accuracy of the procedure for lung cancer diagnosis and staging, and evaluation of the pathological and molecular studies (EGFR, ALK, and ROS1) obtained through EBUS-TBNA and rapid on-site evaluation (ROSE).

(Rev Med Chile 2017; 145: 1165-1171)

Key words: Interventional; Lung Neoplasms; Mediastinum; Neoplasm Staging; Ultrasonography.

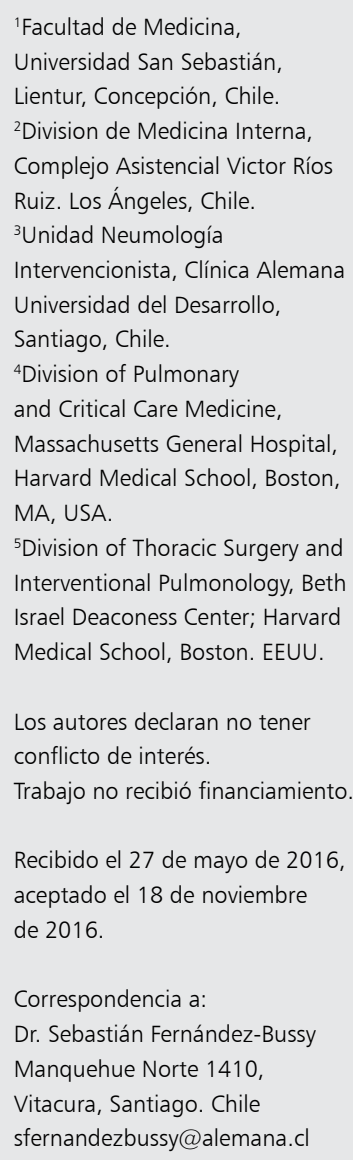

Correspondencia a:

Dr. Sebastián Fernández-Bussy

Manquehue Norte 1410,

Vitacura, Santiago. Chile

sfernandezbussy@alemana.cl

E enfrentamiento diagnóstico y la etapificación de las lesiones sugerentes de cáncer pulmonar, en especial del cáncer pulmonar no células pequeñas (CPNCP), ha presentado un importante desarrollo durante la última década ${ }^{1}$. Clásicamente, la obtención de material anatomopatológico mediante cirugía en sus distintas técnicas (mediastinoscopia, videotoracoscopia disección cervical, entre otras) fue considerada como el estándar de referencia tanto para diagnóstico como para la etapificación mediastínica ${ }^{1,2}$.

Sin embargo, el desarrollo de técnicas mínimamente invasivas como la broncoscopia flexible y posteriormente desarrollo del ultrasonido endobronquial (EBUS), son alternativas que permiten ser una guía endoscópica para la obtención de muestras mediantes biopsia transbronquial (en el caso de la broncoscopia flexible) o aspiración por aguja fina en el caso de EBUS. Estos métodos permiten obtener muestras en forma ambulatoria, con una menor tasa de complicaciones y con resultados variables según la ubicación de la lesión, el tamaño y características estructurales ${ }^{2,3}$.

Existen dos opciones de EBUS, el lineal y radial. El lineal es efectivo en el estudio de lesiones contiguas a la vía aérea central, y permite visualizar linfonodos o lesiones desde los $5 \mathrm{~mm}$ de diámetro ${ }^{4}$. Es el método de elección en la etapificación del mediastino ${ }^{5}$. Actualmente el radial se utilizada para evaluar las capas de la pared de la vía aérea central, diferenciando entre invasión y compresión tumoral: como también para guiar la toma de biopsias de lesiones pulmonares periféricas ${ }^{4}$.

El objetivo del presente capítulo es dar a conocer las indicaciones y el rendimiento diagnóstico del EBUS en el estudio de lesiones sugerentes de cáncer pulmonar, así como su rendimiento en etapificación mediastínica. Además, se abordarán 
aspectos prácticos para el estudio citológico de las muestras obtenidas.

\section{EBUS lineal (L-EBUS)}

Este método se comenzó a utilizar a principios del año 2000 por Herth y cols, y desde entonces se ha utilizado progresivamente para guiar la aspiración transbronquial (TBNA), acumulándose en la actualidad una importante experiencia de más de 10 años ${ }^{4,6,7}$. El EBUS lineal es un broncoscopio específico, donde en su extremo distal posee un transductor de ultrasonido, con emisión de frecuencias de onda entre 5 a $7.5 \mathrm{MHz}$ con mayor penetración a los tejidos subyacentes aunque con menor poder de definición, permitiendo un campo visual de 50 a 90 grados (Figura 1). Se pueden identificar y puncionar en tiempo real diferentes estaciones ganglionares (Figura 2), como las paratraqueales superiores e inferiores, pretraqueales, subcarinales, hiliares e interlobares (estaciones 2R, 2L, 3, 4R, 4L, 7, 10R, 10L, 11R, 11L) correspondientes a la clasificación mediastínica de IASCL. Esto es de gran utilidad en la etapificación del mediastino en cáncer pulmonar. También permite acceder a cualquier lesión adyacente a la tráquea y bronquios principales. Además cuenta con Eco-Doppler que permite diferenciar estructuras vasculares(Figura 3$)^{2,5,8}$.

\section{EBUS Radial (R-EBUS)}

El ultrasonido radial desarrollado en 1990, permite a través de un transductor rotatorio que se introduce por el canal de trabajo del broncoscopio una imagen de 360 grados. Emite una frecuencia entre 12 a $20 \mathrm{MHz}$, lo que permite mejor definición de la anatomía de la pared traqueal y bronquial, aunque con menor poder de penetración a los tejidos subyacentes. Este puede ser utilizado para la evaluación de lesiones centrales de la vía aérea y lesiones periféricas. El ultrasonido radial permite definir las diferentes capas de la pared de tráquea y los bronquios. La capa más interna es la imagen compuesta por el balón y la mucosa (hiperecoica). La segunda capa es la submucosa (hipoecoica). La tercera y la quinta capa corresponden a la capa interna y externa del cartílago (hiperecoicas), separadas por una capa espongiforme (hipoecoica). Esta diferenciación es útil al momento de identificar estructuras comprometidas porinvasión tumoral.

El rendimiento diagnóstico de R-EBUS en el caso de las lesiones pulmonares periféricas puede ser incrementado al combinarse con otras técnicas, como la fluoroscopia, la navegación electromagnética y la broncoscopia virtual. En este escenario, el rendimiento diagnóstico varía entre el 65 al 90\%, disminuyendo considerablemente en lesiones menores de $15 \mathrm{~mm}^{9}$.

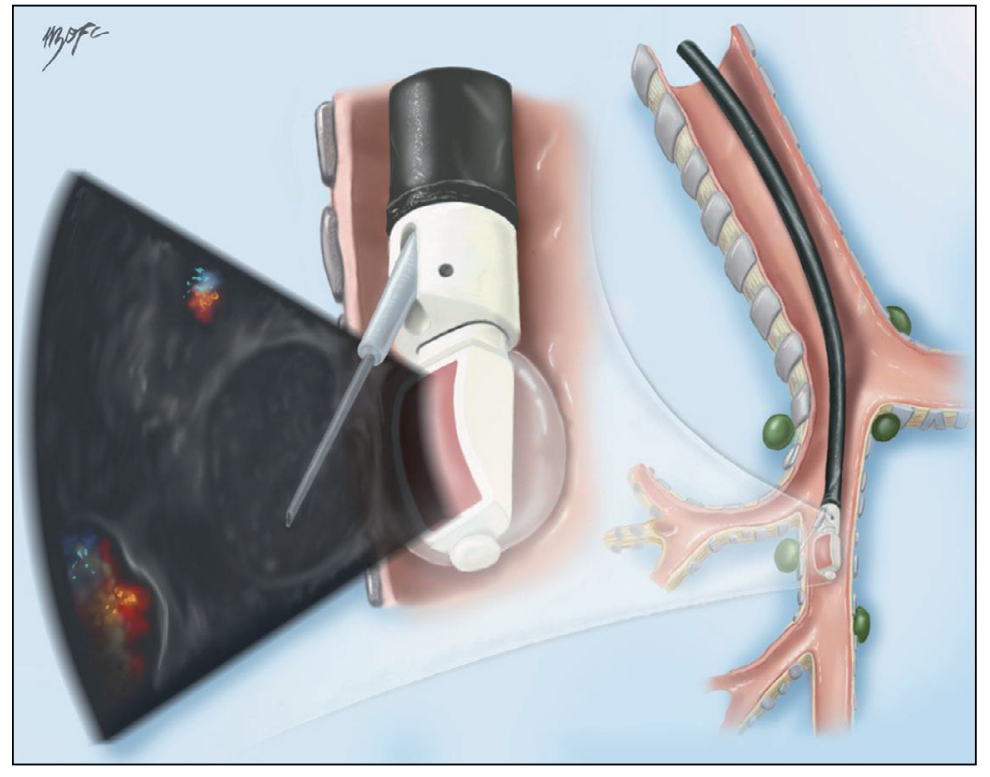

Figura 1. Endosonógrafo bronquial (EBUS) en la etapificación del mediastino y biopsias de lesiones adyacentes a la vía aérea. 


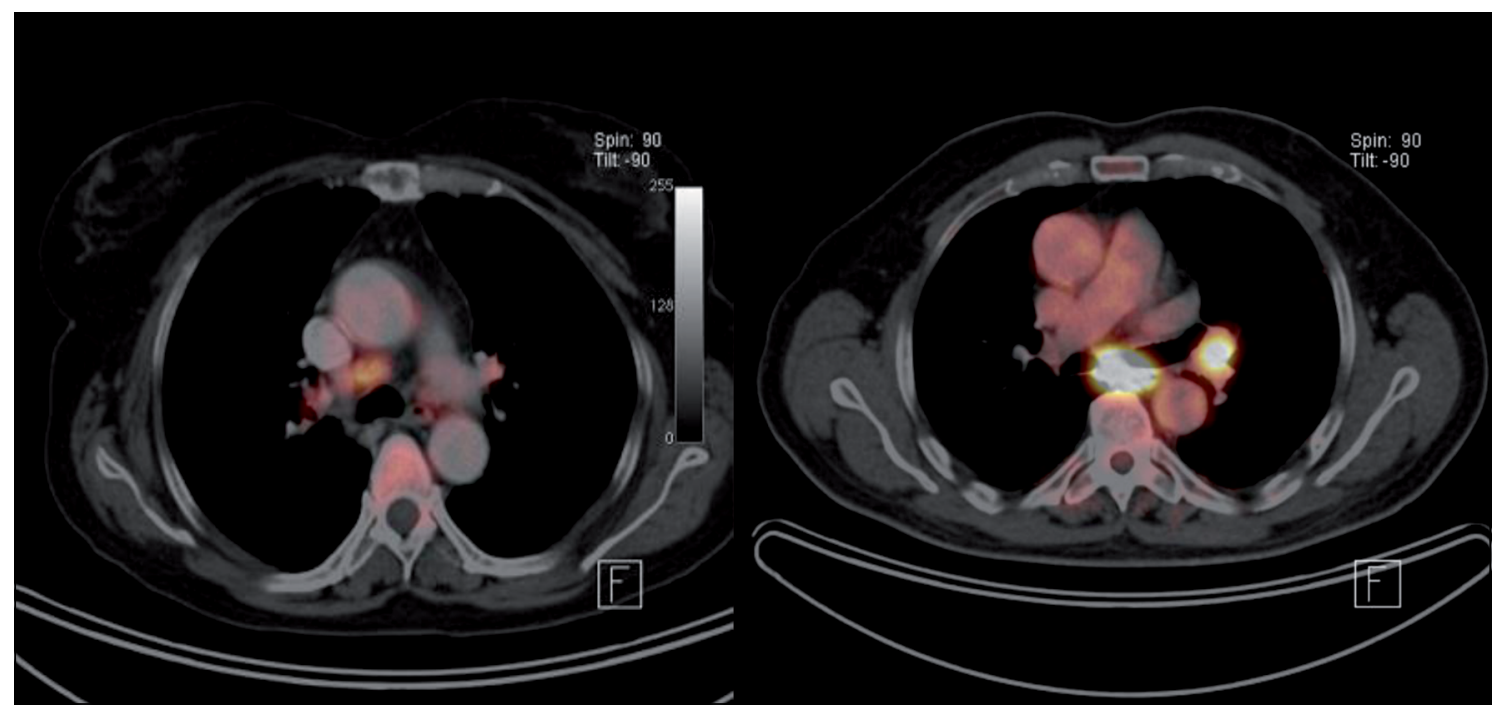

Figura 2. $P E T / C T$ scan con aumento de captación en adenopatía pretraqueal derecha (4R) y subcarinal (7). Biopsia por EBUS.

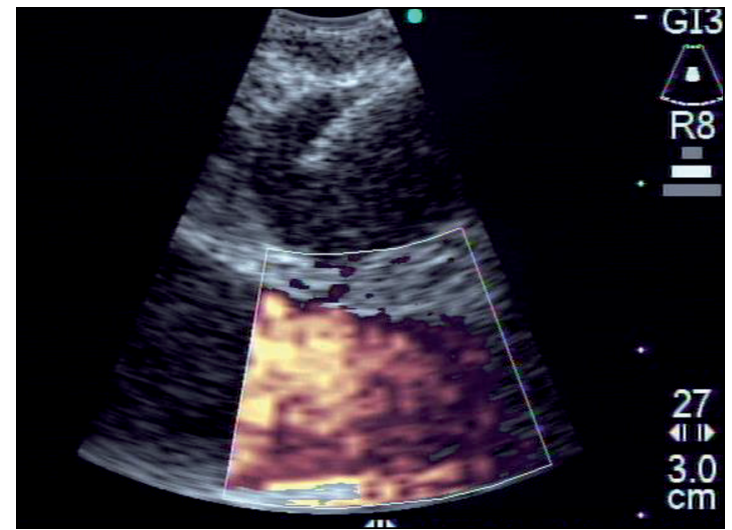

Figura 3. Imagen ecográfica de punción de adenopatía mediastínica por EBUS. Capacidad Doppler.

Respecto a la seguridad de R-EBUS y L-EBUS, ambas técnicas son seguras. Dentro de las complicaciones reportadas en la literatura, la más frecuente es el sangrado auto limitado, con tasas reportadas menores al 5\%. Existen escasos reportes de neumotórax, neumomediastino o infecciones con un porcentaje de eventos fatales de $0,02 \%$.En una serie de 206 pacientes, hubo 3 pacientes con neumotórax y 7 con sangrado mínimo del sitio de biopsia. En otro estudio publicado por nuestro grupo, en el que se incluyeron 354 biopsias en 145 pacientes, encontramos un caso de neumomediastino, de manejo médico, sin presentar otras complicaciones. En una revisión sistemática y meta-análisis, evaluando la seguridad del procedimiento, se incluyeron 190 estudios con un total de 16.181 pacientes. No se encontraron reportes de mortalidad asociada al procedimiento, en 23 pacientes $(0,14 \%)$ se reportaron eventos serios (laceración bronquial, neumotórax, infección, hemorragia con requerimiento de transfusión), mientras que en 35 pacientes se reportaron eventos adversos no serios $(0,22 \%)$. Sin embargo, estas complicaciones fueron reportadas con mayor frecuencia en pacientes con lesiones quísticas y sarcoidosis, en el caso de cáncer, estas fueron infrecuentes ${ }^{3}$.

\section{Diagnóstico de lesiones sugerentes de cáncer pulmonar}

El rendimiento en lesiones centrales mediante L-EBUS es alto y debe ser indicado como método diagnóstico en aquellos pacientes en los que la realización de broncoscopia flexible con biopsia trans-bronquial no es factible o en aquellas lesiones con diámetro menor de $20 \mathrm{~mm}$. La visualización de la lesión mediante el ultrasonido es el factor más significativo para obtener una muestra 
diagnóstica, está demostrado que se deben tomar un mínimo de 5 biopsias para obtener una muestra representativa ${ }^{8}$.

En el caso de R-EBUS para estudio de lesiones periféricas, en un metaanálisis de Steinford y cols, donde se incluyeron 16 estudios con un total de 1.420 pacientes, encontraron una sensibilidad o rendimiento diagnóstico de 73\% (IC: 0,70-0,76), esta variaba desde 49 a $88 \%$ en los distintos estudios, no relacionándose con algunos dispositivos adicionales usados en algunos centros, sino que más bien el rendimiento aumentaba claramente al ubicar la lesión con el R-EBUS, con una especificidad de $100 \%$, con un $\operatorname{LR}(+)$ de 26,8 y un LR (-) de 0,28. En este trabajo hubo10 estudios que reportaron el rendimiento diagnóstico según el tamaño de la lesión, con $56,3 \%$ en lesiones $\leq 2 \mathrm{~cm}$ y $77,7 \%>2 \mathrm{~cm}$. Un importante hallazgo es que la sensibilidad del estudio diagnóstico se correlaciona claramente con la prevalencia de enfermedad neoplásica ${ }^{10}$.

A nivel local, se encuentra reportada el rendimiento diagnóstico de EBUS para lesiones adyacentes a la vía aérea central, de un total de 128 pacientes incluidos durante un período de 3 años, se encontró una sensibilidad de $96,7 \%$ y especificidad de $100 \%$ con un valor predictivo negativo de 93,3\%. El diámetro promedio de estas lesiones fue de $18,53 \mathrm{~mm}$, siendo punciones en su mayoría en los segmentos $4 \mathrm{R}$ y 7 de la clasificación IASLC ${ }^{11}$.

\section{Etapificación de mediastino mediante EBUS-TBNA}

La etapificación mediastínica es relevante al momento de evaluar un paciente con diagnóstico de cáncer pulmonar, o al momento de realizar unare- etapificación posterior al tratamiento. El uso del PET-CT como método de etapificación es insuficiente al momento de establecer compromiso nodal N2/N3. En una revisión Cochrane, donde se incluyeron 45 estudios, se obtuvo una sensibilidad mediante un aumento de la actividad SUVmax $\geq 2,5$ de $81,3 \%$ (IC $70,2 \%-88,9 \%$ ) y especificidad de 79,4\% (IC 70\%-86,5\%) $)^{12}$.

Tanto en el caso de etapificación como re-etapificación, la aplicación de EBUS-TBNA es de alto rendimiento diagnóstico y tanto las guías de las sociedades americanas de la ACCP (American
College of ChestPhysicians) como las europeas lo recomiendan como el método de primera elección ${ }^{2,5}$.

El rendimiento descrito en etapificación presenta una sensibilidad por sobre $90 \%$ con una especificidad de $100 \%$.En una revisión sistemática y meta-análisis, donde se incluyeron 11 estudios con 1.229 pacientes con indicación de EBUS para etapificación mediastínica secundario a cáncer pulmonar no células pequeñas, se encontró una sensibilidad de 93\% (95\% CI, 91\%-94\%) y una especificidad de $100 \%$ (95\% CI, 99\%-100\%) $)^{13}$. Sin embargo, este método diagnóstico no es suficiente para localizar todas las estaciones ganglionares mediastínica, en especial las estaciones 5, 6, 8 y 9 del mapa de estaciones ganglionares mediastínica definidas por la IASLC. En estos casos se puede complementar la técnica con endoscopia guiada por ultrasonido (EUS) ${ }^{14}$. Esta combinación, reporta un rendimiento comparado con etapificación mediante el empleo de técnicas quirúrgicas de $86 \%$ (IC 0,82-0,90); especificidad 100 (IC 99100); likelihood ratio (LR) (+) de 51,77 y LR (-) de $0,15^{15,16}$.

En un estudio clínico aleatorizado publicado recientemente, se comparó el rendimiento de este procedimiento como primera alternativa de estudio contra las técnicas quirúrgicas habituales. Como resultado se obtuvo que la estrategia de estudio inicial mediante EBUS disminuye en dos semanas el tiempo entre el estudio y la toma de decisión clínica, comparada con el protocolo de estudio con imágenes y posterior cirugía. Por otro lado, se obtuvo una menor incidencia de toracotomías innecesarias, sin encontrar impacto en la mortalidad. En este estudio EBUS-TBNA presentó una sensibilidad de $92 \%$ y especificidad de $100 \%{ }^{17}$. En otro estudio de costo efectividad, basado en un estudio clínico aleatorizado publicado en el año 2010, concluyen que el uso de EBUS como estrategia de etapificacion mediastínica inicial, seguido pormediastinoscopia en los casos de presentar EBUS-TBNA negativo, tiene una sensibilidad de 94\% para detectar compromiso metastásico N2/ N3 (IC 85-98\%), comparado conla estrategia de mediastinoscopia como primera opción que reporta 79\% (IC 66-88\%). Además, se asocia a una disminución de toracotomías innecesarias y mejor calidad de vida durante la etapificación ${ }^{18}$.

En otra publicación, nuestro grupo evaluó el rendimiento diagnóstico de EBUS lineal para la 
etapificación mediastínica en pacientes con diagnóstico o sospecha de cáncer pulmonar, encontrando una sensibilidad de $91,17 \%$, especificidad de $100 \%$ y valor predictivo negativo de $92,9 \%{ }^{19}$.

En el caso de los pacientes con indicación de re-etapificación mediastínica, el rendimiento de EBUS-TBNA, presenta una sensibilidad de 64\%, especificidad de $100 \%$, valor predictivo positivo de $100 \%$ y valor predictivo negativo de $82 \%{ }^{20}$.

\section{Rendimiento citológico de muestras mediante EBUS-TBNA}

Al momento de realizar EBUS-TBNA, se obtienen un mínimo de 5 muestras por aspiración, que se colocan en formalina y son procesadas como un block celular. Estas muestras son suficientes para realizar técnicas inmunohistoquímicas y análisis de marcadores moleculares. En un estudio se incluyeron 92 punciones mediante EBUS-EUS con biopsias simultáneas encontrando una concordancia citología-biopsia para subtipificación de CPNCP de 76\% (кappa test $=0,52$ ), en casos en que existió bloque celular fue $96 \%$ (карpa test $=0,91$ ) vs $69 \%$ (Kappa test $=0,39$ ) para casos $\sin$ bloque $^{21}$. En otra serie de 2 centros de Inglaterra, la precisión con bloque celular fue de $64 \%$ y al agregar inmunohistoquímica (IHQ) aumentó a $84 \%$. La incorporación de IHQ en bloques celulares mejoró la exactitud diagnóstica para ADC de $56 \%$ a $83,2 \%{ }^{22,23}$.

En otra serie de muestras apareadas en un mismo procedimiento, la precisión se determinó por resección o autopsia $(\mathrm{n}=21)$ o IHQ $(\mathrm{n}=43)$. Las categorías definitiva, probable o no clasificable fueron similares para biopsia $(71 \%, 23 \%$ y $6 \%$, respectivamente) y citología $(69 \%, 19 \%$ y $12 \%), p=0,29$. La concordancia para subtipos de CPNCP entre biopsia y citología fue $93 \%$. El $\kappa$ para acuerdo fue 0,88 (IC95\% 0,60-0,89) para ADC y 0,76 (IC95\% 0,63-0,89) para CE. Todos los diagnósticos concordantes verificados fueron correctos $(\mathrm{n}=57)^{24}$.

Respecto al estudio de mutaciones mediante técnicas de biología molecular, en especial en pacientes en los que se busca confirmar la presencia de mutaciones como EGFR, ALK y ROS1, se evaluó la presencia de mutación EGFR, en 62 muestras apareadas, existió $91,7 \%$ de concordancia cito-histológica. Esta correlación fue óptima
(100\%) cuando la concentración de DNA > $25 \mathrm{ng} /$ $\mu \mathrm{l},>30$ células tumorales, o tumor $>30 \%$ por lo que se puede concluir que las muestras obtenidas mediante EBUS-TBNA son suficientes para establecer la presencia de mutaciones ${ }^{25,26}$. En un estudio publicado recientemente por nuestro grupo, donde se incluyeron 86 pacientes para estudio con marcadores moleculares, encontramos $97,67 \%$ de muestras con tejido "suficiente" para realizar estudio de EGFR, 94,2\% para estudio de ALK y $83,33 \%$ para ROS1 ${ }^{27}$.

Finalmente, en algunos centros se aplica como técnica complementaria a EBUS-TBNA, el apoyo mediante citología in situ (ROSE). Esta modificación a la técnica original consiste en la obtención de una muestra citológica con estudio ultra rápido, mediante visualización directa al microscopio óptico por un especialista. Este complemento ha sido estudiado principalmente en lesiones pulmonares hiliares o mediastínica con L- EBUS en donde no se ha demostrado un aumento en el rendimiento diagnóstico, por lo que en nuestro centro no se realiza esta técnica de rutina ${ }^{28}$.

\section{Conclusión}

Considerando que más del 75\% de los pacientes con diagnóstico de reciente de cáncer pulmonar no son pasibles de tratamiento quirúrgico, una alternativa para poder realizar el diagnóstico y etapificación es el uso de son técnicas mínimamente invasivas. En ese sentido, el ultrasonido endobronquial (EBUS) ofrece esta posibilidad, con sensibilidad diagnóstica comparable a los métodos quirúrgicos. Ademas, el EBUS es el procedimiento recomendado por las guías del American College of Chest Physicians (ACCP), European Respiratory Society (ERS), European Thoracic Society (ETS) y European Society for Medical Oncology (ESMO), para la etapificación del mediastino en el cáncer pulmonar.

\section{Referencias}

1. Vilmann P, Puri R. The complete "medical" mediastinoscopy (EUS-FNA + EBUS-TBNA). Minerva medica 2007; 98: 331-8.

2. Silvestri GA, González AV, Jantz MA, Margolis ML, Gould MK, Tanoue LT, et al. Methods for staging 
non-small cell lung cancer: Diagnosis and management of lung cancer, 3rd ed: American College of Chest Physicians evidence-based clinical practice guidelines. Chest 2013; 143: e211S-50S.

3. von Bartheld MB, van Breda A, Annema JT. Complication rate of endosonography (endobronchial and endoscopic ultrasound): a systematic review. Respiration; international review of thoracic diseases 2014; 87: 343-51.

4. Zaric B, Eberhardt R, Herth F, Stojsic V, Carapic V, Popovic ZP, et al. Linear and radial endobronchial ultrasound in diagnosis and staging of lung cancer. Expert Rev Med Devices 2013; 10: 685-95.

5. Vilmann P, Clementsen PF, Colella S, Siemsen M, De Leyn P, Dumonceau JM, et al. Combined endobronchial and esophageal endosonography for the diagnosis and staging of lung cancer: European Society of Gastrointestinal Endoscopy (ESGE) Guideline, in cooperation with the European Respiratory Society (ERS) and the European Society of Thoracic Surgeons (ESTS). Endoscopy 2015; 47: 545-59.

6. Herth F, Becker HD. Endobronchial ultrasound of the airways and the mediastinum. Monaldi Arch Chest Dis 2000; 55: 36-44.

7. Rintoul RC, Ahmed R, Dougherty B, Carroll NR. Linear endobronchial ultrasonography: a novelty turned necessity for mediastinal nodal assessment. Thorax 2015; 70 : 175-80.

8. Rivera MP, Mehta AC, Wahidi MM. Establishing the diagnosis of lung cancer: Diagnosis and management of lung cancer, 3rd ed: American College of Chest Physicians evidence-based clinical practice guidelines. Chest 2013; 143: e142S-65S.

9. Gompelmann D, Herth FJ. Role of endobronchial and endoscopic ultrasound in pulmonary medicine. Respiration; international review of thoracic diseases 2014; 87 : 3-8.

10. Steinfort DP, Khor YH, Manser RL, Irving LB. Radial probe endobronchial ultrasound for the diagnosis of peripheral lung cancer: systematic review and meta-analysis. The European respiratory journal 2011; 37: 902-10.

11. Fernández-Bussy S, Labarca G, Caviedes I, Folch E, Majid A. Endobronchial ultrasound and fine needle aspiration for central airway lesions. Rev Med Chile 2016; 144: 341-6.

12. Schmidt-Hansen M, Baldwin DR, Hasler E, Zamora J, Abraira V, Roque IFM. PET-CT for assessing mediastinal lymph node involvement in patients with suspected resectable non-small cell lung cancer. The Cochrane database of systematic reviews 2014; 11: CD009519.

13. Gu P, Zhao YZ, Jiang LY, Zhang W, Xin Y, Han BH.
Endobronchial ultrasound-guided transbronchial needle aspiration for staging of lung cancer: a systematic review and meta-analysis. European journal of cancer 2009; 45 : 1389-96.

14. Vilmann P, Krasnik M, Larsen SS, Jacobsen GK, Clementsen P. Transesophageal endoscopic ultrasound-guided fine-needle aspiration (EUS-FNA) and endobronchial ultrasound-guided transbronchial needle aspiration (EBUS-TBNA) biopsy: a combined approach in the evaluation of mediastinal lesions. Endoscopy 2005; 37: 833-9.

15. Zhang R, Ying K, Shi L, Zhang L, Zhou L. Combined endobronchial and endoscopic ultrasound-guided fine needle aspiration for mediastinal lymph node staging of lung cancer: a meta-analysis. Eur J Cancer 2013; 49: 1860-7.

16. Labarca GAC, Ortega F, Arenas A, Majid A, Folch E, Mehta HJ, et al. Minimally Invasive Methods for Staging in Lung Cancer: Systematic Review and Meta-Analysis. Pulmonary Medicine 2016; 2016: 1024709.

17. Navani N, Nankivell M, Lawrence DR, Lock S, Makker $\mathrm{H}$, Baldwin DR, et al. Lung cancer diagnosis and staging with endobronchial ultrasound-guided transbronchial needle aspiration compared with conventional approaches: an open-label, pragmatic, randomised controlled trial. Lancet Respir Med 2015; 3: 282-9.

18. Sharples LD, Jackson C, Wheaton E, Griffith G, Annema JT, Dooms C, et al. Clinical effectiveness and cost-effectiveness of endobronchial and endoscopic ultrasound relative to surgical staging in potentially resectable lung cancer: results from the ASTER randomised controlled trial. Health Technol Assess 2012; 16: 1-75, iii-iv.

19. Fernández-Bussy S, Labarca G, Canals S, Caviedes I, Folch E, Majid A. Diagnostic yield of endobronchial ultrasound-guided transbronchial needle aspiration for mediastinal staging in lung cancer. Jornal brasileiro de pneumologia : publicacao oficial da Sociedade Brasileira de Pneumologia e Tisilogia 2015; 41: 219-24.

20. Zielinski M, Szlubowski A, Kolodziej M, Orzechowski S, Laczynska E, Pankowski J, et al. Comparison of endobronchial ultrasound and/or endoesophageal ultrasound with transcervical extended mediastinal lymphadenectomy for staging and restaging of non-small-cell lung cancer. J Thorac Oncol 2013; 8: 630-6.

21. Tournoy KG, Carprieaux M, Deschepper E, van Meerbeeck JP, Praet M. Are EUS-FNA and EBUS-TBNA specimens reliable for subtyping non-small cell lung cancer? Lung cancer 2012; 76: 46-50.

22. Ocque R, Tochigi N, Ohori NP, Dacic S. Usefulness of immunohistochemical and histochemical studies in the classification of lung adenocarcinoma and squamous 
cell carcinoma in cytologic specimens. American journal of clinical pathology 2011; 136: 81-7.

23. Wallace WA, Rassl DM. Accuracy of cell typing in nonsmall cell lung cancer by EBUS/EUS-FNA cytological samples. The European respiratory journal 2011; 38: 911-7.

24. Sigel CS, Moreira AL, Travis WD, Zakowski MF, Thornton RH, Riely GJ, et al. Subtyping of non-small cell lung carcinoma: a comparison of small biopsy and cytology specimens. J Thorac Oncol 2011; 6: 1849-56.

25. Sun PL, Jin Y, Kim H, Lee CT, Jheon S, Chung JH. High concordance of EGFR mutation status between histologic and corresponding cytologic specimens of lung adenocarcinomas. Cancer cytopathology 2013; 121: 311-9.

26. Navani N, Brown JM, Nankivell M, Woolhouse I,
Harrison RN, Jeebun V, et al. Suitability of endobronchial ultrasound-guided transbronchial needle aspiration specimens for subtyping and genotyping of non-small cell lung cancer: a multicenter study of 774 patients. American journal of respiratory and critical care medicine 2012; 185: 1316-22.

27. Fernández-Bussy S LG, Pires Y, Caviedes I, Burotto M. Análisis moleculares de EGFR, mutación de resistencia al EGFR, ALK y ROS1 en muestras obtenidas mediante PATB-USEB en Chile. Arch Bronconeumol 2016; En prensa.

28. Joseph M, Jones T, Lutterbie Y, Maygarden SJ, Feins RH, Haithcock BE, et al. Rapid on-site pathologic evaluation does not increase the efficacy of endobronchial ultrasonographic biopsy for mediastinal staging. Ann Thorac Surg 2013; 96: 403-10. 